\title{
AMERICAN ACADEMY OF FAMILY PHYSICIANS
}

AAFP Reprint No. 282

Recommended Curriculum Guidelines for Family Medicine Residents

\section{Women's Health and Gynecologic Care}

This document was endorsed by the American Academy of Family Physicians (AAFP) to be used in conjunction with the recommended AAFP Curriculum Guideline No. 261 Maternity Care.

\section{Introduction}

This AAFP Curriculum Guideline defines a recommended training strategy for family medicine residents. Attitudes, behaviors, knowledge, and skills that are critical to family medicine should be attained through longitudinal experience that promotes educational competencies defined by the Accreditation Council for Graduate Medical Education (ACGME), www.acgme.org. The family medicine curriculum must include structured experience in several specified areas. Much of the resident's knowledge will be gained by caring for ambulatory patients who visit the family medicine center, although additional experience gained in various other settings (e.g., an inpatient setting, a patient's home, a long-term care facility, the emergency department, the community) is critical for well-rounded residency training. The residents should be able to develop a skillset and apply their skills appropriately to all patient care settings.

Structured didactic lectures, conferences, journal clubs, and workshops must be included in the curriculum to supplement experiential learning, with an emphasis on outcomes-oriented, evidence-based studies that delineate common diseases affecting patients of all ages. Patient-centered care, and targeted techniques of health promotion and disease prevention are hallmarks of family medicine and should be integrated in all settings. Appropriate referral patterns, transitions of care, and the provision of costeffective care should also be part of the curriculum. 
Program requirements specific to family medicine residencies may be found on the ACGME website. Current AAFP Curriculum Guidelines may be found online at www. aafp.org/cg. These guidelines are periodically updated and endorsed by the AAFP and, in many instances, other specialty societies, as indicated on each guideline.

Please note that the term "manage" occurs frequently in AAFP Curriculum Guidelines. "Manage" is used in a broad sense indicating that the family physician takes responsibility that optimal and complete care is provided to the patient. To manage does not necessarily mean that all aspects of care need to be directly delivered personally by the family physician and may include appropriate referral to other health care providers, including other specialists for evaluation and treatment.

Each residency program is responsible for its own curriculum. This guideline provides a useful strategy to help residency programs form their curricula for educating family physicians.

\section{Preamble}

While we recognize a non-binary gender spectrum, this curriculum guideline addresses care of patients of the female sex. Issues specifically related to caring for transgender patients can be found under the LGBT curriculum guidelines. For the sake of clarity, the words woman and women's health should be assumed to be synonymous with individuals of female sex.

Women's health care addresses the unique, multidisciplinary aspects of issues affecting women. In providing a wide range of medical services, the family physician can provide preventive and wellness care, diagnose general medical illnesses and disease processes unique to women, and care for women and their families. The goal of these guidelines is to familiarize the family medicine resident with attitudes, knowledge, and skills that are important for the care of women and their families.

Family physicians must be trained to care for women throughout the life cycle and must appreciate challenges such as adolescence, sexuality, family planning, balance of family life and career, and aging within the female patient's culture. Health promotionincluding screening, counseling, and vaccination-is a foundation of family medicine. For the majority of their reproductive lives, most women try to prevent pregnancy, so we highlight this aspect of care.

The psychological and physiologic changes of menarche, contraception, pregnancy, lactation, and menopause impact women in many aspects of their lives, requiring clinical skills on the family physician's part to provide education, diagnostic testing, treatment, and appropriate referral that is safe and effective. Women are living to an advanced age more frequently than their male counterparts, therefore cognitive, affective, and functional assessments, as well as end-of-life discussions, are important aspects of care. 
This AAFP Curriculum Guideline provides an outline of the attitudes, knowledge, and skills that family physicians should attain during residency training to provide high quality care to their female patients. See also AAFP Curriculum Guideline No. 261 Maternity Care.

\section{Competencies}

At the completion of residency training, a family medicine resident should be able to:

- Diagnose and develop treatment plans for common conditions affecting female patients at different stages throughout the reproductive lifespan (Medical Knowledge, Systems-based Practice, Practice-based Learning and Improvement)

- Perform appropriate preventive services, including screening tests and wellness counseling based on the patient's age and risk factors (Patient Care, Medical Knowledge)

- Perform routine gynecologic procedures, including, but not limited to, pap smear, endometrial biopsy, collection and interpretation of vaginal and cervical samples, and placement of long-acting reversible contraception (LARC) (Patient Care, Medical Knowledge)

- Offer patient-centered comprehensive contraceptive counseling and options, including long-acting reversible contraception (LARC) (Patient Care, Medical Knowledge)

- Understand the risks and appropriately counsel patients about non-gynecologic medical problems that may manifest differently or more frequently in women, including heart disease, stroke, osteoporosis, anxiety/depression, and intimate partner violence (Patient Care, Medical Knowledge)

- Consult with obstetrician-gynecologists (OB-GYNs), other physician specialists, and allied health care professionals to provide optimal health services for women (Medical Knowledge, Systems-based Practice)

- Communicate respectfully and effectively with women of all ages to act as a patient advocate and coordinator of care for female patients across the continuum of outpatient, inpatient, and assisted care (Systems-based Practice)

\section{Attitudes and Behaviors}

The resident should develop attitudes and behaviors that encompass:

- A caring, compassionate, and respectful approach to the female patient's role as an informed participant in her own health care decisions and those affecting her family 
- Recognition of the need to empower the female patient in the decision-making process and provide information to enable the female patient to make informed decisions

- Recognition that a woman's health is affected not only by medical problems, but also by family, career, life cycle, relationships, and community

- Appreciation of the role that many women play in the health of the family by selecting health care professionals, providing family care, and making lifestyle decisions for the family

- Awareness of the effects of public perception and media representation of women and body image on female patients

- Awareness of implicit bias, particularly in relationship to race and ethnicity

- Awareness of issues facing heterosexual, lesbian, bisexual, and transgender patients (See AAFP Curriculum Guideline No. 289D - Lesbian, Gay, Bisexual, Transgender Health)

- Awareness of the widespread and complex health effects of physical, emotional, and sexual abuse on women

- Awareness of the issues of female circumcision/female genital mutilation when caring for females from cultures that carry out such practices

\section{Knowledge}

In the appropriate setting, the resident should demonstrate the ability to apply knowledge of (See also AAFP Curriculum Guideline No. 261 - Maternity Care):

1. Health promotion, disease prevention, and periodic health evaluation

a. Basic aspects of normal growth and development of females from puberty to adulthood (and variants of normal)

b. Normal physiology of reproduction in healthy women from puberty to menopause

c. Normal physiological sexual responses and diagnosis of sexual dysfunction (including initial treatment and referral to appropriate resources)

d. Cervical dysplasia screening guidelines (including HPV), colposcopic evaluation, biopsy, treatment, and referral

e. Appropriate evaluation and counseling using evidence-based guidelines for:

i. Nutritional needs through the female life cycle

ii. Cancer screening guidelines

iii. Vaccination

iv. Exercise prescription

v. Osteoporosis prevention and treatment

vi. Smoking cessation

vii. Weight management 
viii. Risks and unique presentations of cardiovascular disease in women (including appropriate testing and treatment strategies for symptomatic women)

ix. Mental health and substance abuse screening recommendations

f. Women's unique risks in the community (including poverty, violence, access to health care for pregnant and non-pregnant women, and teen pregnancy) and the impact of these factors on infant morbidity and mortality

g. Basic understanding of complementary/integrative therapies

2. Menstruation

a. Physiology of puberty, menarche, and menstrual cycles, including normal variations

b. Evaluation and treatment for conditions of abnormal menstruation

i. Amenorrhea: evaluation and management of both primary and secondary etiologies

ii. Abnormal uterine bleeding

iii. Postcoital bleeding

iv. Dysmenorrhea

3. Family planning

a. Preconception counseling for women of all reproductive age groups

b. Appropriate evaluation and counseling using evidence-based guidelines for contraception for women in all reproductive age groups

i. Permanent

ii. Reversible

1) Oral

2) Injectable

3) Patches

4) Intravaginal contraceptive ring

5) Long-acting reversible contraception (LARC)

a) Intrauterine devices (IUDs)

b) Implants

6) Natural family planning

7) Barrier methods

8) Postcoital (emergency) contraception

c. Etiologies of female infertility, as well as a family-centered approach to evaluation, testing, counseling, and referral resources (including counseling regarding assisted reproductive technology and adoption)

4. Early pregnancy evaluation and management

a. Dating of early pregnancy

b. Counseling for unintended pregnancy, including options of adoption, abortion (medication and aspiration), and continuing the pregnancy to term 
c. Assessment and management of first trimester bleeding, including ectopic pregnancy diagnosis and management

d. Assessment and management of early pregnancy loss, including expectant, medication, and aspiration options

e. Assessment and management of post-miscarriage and post-abortion symptoms and complications, including mental health implications

5. Infertility

a. Diagnosis of infertility and appropriate referral.

6. Family-centered maternity care (See also AAFP Curriculum Guideline No. 261 Maternity Care)

7. General gynecologic pathology

a. Benign and malignant neoplasms of the external and internal genitalia

b. Uterine and adnexal pathology, evaluation, treatment and appropriate referral: fibroids, endometrial hyperplasia, postmenopausal vaginal bleeding, malignant uterine lesions, and adnexal masses.

c. Pelvic pain: evaluation and differential diagnosis of acute and chronic pelvic pain, including recognition of emergencies (e.g., ovarian torsion), awareness of association between historical or ongoing sexual or domestic abuse, and indications for referral (e.g., infection, endometriosis, tumors)

d. Polycystic ovary syndrome: presenting symptoms, evaluation and initial treatment, as well as association with type 2 diabetes mellitus

e. Female sexual dysfunction: evaluation, counseling, and management, including problems of libido, dyspareunia, and anorgasmia

f. Trauma: patient-centered, sensitive evaluation of both accidental trauma to the genital region and trauma in victims of intimate partner violence and sexual assault

g. Urogynecology

i. Urinary tract infections (UTIs): diagnosis and management of uncomplicated acute UTI, as well as recurrent or complicated UTI; indications for and management of prophylactic antibiotics

ii. Incontinence: screening, evaluation, and treatment options for stress incontinence and overactive bladder, including medications, pelvic floor therapies, behavioral modifications, and referral for surgery

iii. Interstitial cystitis: presenting symptoms, evaluation, management, and referral

iv. Pelvic organ prolapse (POP): recognition, diagnosis, management and referral

8. Infections of the genital tract 
a. Sexually transmitted infections, cervicitis, and pelvic inflammatory disease: epidemiology, screening, presentation, evaluation and treatment (outpatient versus inpatient management)

b. Vaginitis: risk factors, presenting symptoms, evaluation, and treatment

c. Risks factors for, screening tests for, and presentations of HIV in women, as well as initial evaluation, counseling, and referral to resources in the community for both pregnant and non-pregnant female patients who have HIV

9. Breast health

a. Anatomy and physiology of benign diseases of the breast (including cysts, adenomas, and fibrocystic changes through the menstrual cycle)

b. Evaluation and management of breast disease, including mastodynia, galactorrhea, and nipple discharge.

c. Counseling and indications for referral for breast reduction surgery

d. Recommendations based on evidence and controversies related to screening for breast cancer using clinical breast examination (CBE), breast self-examinations (BSE), imaging, and genetic testing

e. Initial recommendations for treatment modalities, referral resources, and primary care follow-up for patients who have breast cancer

f. Types, risks, and psychological impact of breast implants

10. Mental health

a. Unique risks and presentations of mental health problems in women, including:

i. Major depressive disorder

ii. Peripartum blues, anxiety, depression, and psychosis

iii. Anxiety disorders and stress management

iv. Problems with self-esteem

v. Eating disorders

vi. Obesity

vii. Alcohol and substance abuse

viii. Chronic pain and disability

b. Physiology and diagnostic criteria of premenstrual syndrome (PMS) and premenstrual dysphoric disorder (PMDD), and available treatments for each

11. Physical, emotional, sexual, and intimate partner violence

a. Epidemiology, risk factors, and red flags for identifying intimate partner violence and resources available to assist affected women and children.

b. Components of the evaluation and treatment of survivors of rape, sexual assault, and sexual harassment (including psychosocial and legal issues as well as mandatory reporting regulations) 
12. Care of the older woman
a. Menopause: diagnosis, physical, emotional, and sexual impact of the transition, risks/benefits of hormone replacement therapy, complementary alternatives
b. Pelvic floor dysfunction: presenting symptoms (urinary incontinence and pelvic floor prolapse), medical and surgical treatment options, appropriate referral
c. Postmenopausal bleeding: workup, management and referral.
d. Cognitive, affective, and functional assessment
e. End of life planning

\section{Skills}

In the appropriate setting, the resident should demonstrate the ability to independently perform the following skills (when this is not available or appropriate, the resident should have exposure to the opportunity to practice these skills):

1. Offering comprehensive contraceptive options
a. Counseling and prescribing for all forms of birth control (including all hormonal and non-hormonal methods as described above)
b. IUD insertion and removal
c. Implantable contraceptive insertion and removal
d. Diaphragm fitting
e. Prescription of emergency contraception
f. Quick-start approach to prescribing contraception, allowing most women with a negative pregnancy test to start a contraceptive method at any point in the menstrual cycle

2. Outpatient gynecologic procedures, interpretation, and appropriate referral
a. Female breast exam, when indicated
b. Gynecologic exam, including atraumatic speculum and bimanual exams
c. Vaginal and cervical cytology collection (with HPV testing, as indicated)
d. Endometrial biopsy
e. Interpretation of urinalysis, vaginal wet mount, $\mathrm{KOH}$ prep, vaginal cultures
f. Vaginal foreign body removal
g. Biopsy of vulvar lesions
h. Cervical polypectomy

3. Counseling 

a. Results of cervical cytology, mammography, osteoporosis screening, sexually transmitted infection screening, and other tests
b. Appropriate referrals
c. Family and relationship stresses
d. Intimate partner and family violence
e. Contraceptive choices
f. Pregnancy options (including adoption, abortion, and parenting)
g. Pregnancy loss, ectopic pregnancy, and molar pregnancy
h. Infertility
i. Healthy lifestyle choices

4. Pregnancy management (See also AAFP Curriculum Guideline No. 261 - Maternity Care)
a. Prenatal counseling about aspects of normal pregnancy, delivery, and family adaptation
b. Evaluation of gestational age and pregnancy risks in early pregnancy
c. Referral for first trimester termination if desired by patient
d. Low-risk prenatal care
e. Identification of high-risk pregnancies

5. Labor and delivery management (See also AAFP Curriculum Guideline No. 261 Maternity Care)

6. Advanced skills in obstetrics and gynecology for family medicine residents who wish to include these in their practices:
a. Colposcopy, cervical biopsy, and endocervical curettage
b. Cervical cryosurgery
c. Bartholin duct cyst management
d. Vulvovaginal biopsy
e. Breast cyst aspiration
f. Loop electrosurgical excision procedure with paracervical block
g. First trimester termination: surgical or medical
h. Bilateral tubal ligation
i. Uterine aspiration for incomplete or missed first trimester abortion
j. Pessary fitting
k. Dilation and curettage 
7. Gynecologic surgery
a. Assisting with common major surgical procedures, including hysterectomy
b. Post-operative management following gynecologic or obstetric surgery

\section{Implementation}

Core cognitive ability and skills require experience in structured rotations in obstetrics and gynecology. Emphasis on the ambulatory care of patients (including counseling, examination, and outpatient procedures) is crucial and can be taught in both continuity clinics and high-volume specialty clinics. Workshops in gynecologic procedures, didactics, and communication seminars can enhance clinical experience.

Faculty role models and family physicians who provide comprehensive reproductive health care should be available to teach residents and observe their interactions with female patients. Residents of both genders should care for an adequate number of female patients of all ages, along with their families, to learn the full spectrum of issues affecting women.

\section{Resources}

Apgar BS, Brotzman GL, Spitzer M. Colposcopy, Principles and Practice. $2^{\text {nd }}$ ed. Philadelphia, Pa.: Saunders; 2008.

Berek JS. Berek and Novak's Gynecology. 15th ed. Philadelphia, Pa.: Lippincott Williams \& Wilkins; 2012.

Carlson KJ, Eisenstat SA, Frigoletto FD, Schiff I. Primary Care of Women. $2^{\text {nd }}$ ed. Philadelphia, Pa.: Mosby; 2002.

Fritz MA, Speroff L. Clinical Gynecologic Endocrinology and Infertility. $8^{\text {th }}$ ed. Philadelphia, Pa.: Lippincott Williams \& Wilkins; 2011.

Gabbe SG, Niebyl JR, Simpson JL, et. al. Obstetrics: Normal and Problem Pregnancies. $7^{\text {th }}$ ed. Philadelphia, Pa.: Elsevier; 2017.

Lentz G, Lobo RA, Gershenson D, Katz VL. Comprehensive Gynecology. $6^{\text {th }}$ ed. Philadelphia, Pa.: Mosby Elsevier; 2012.

Manetta A. Cancer Prevention and Early Diagnosis in Women. Philadelphia, Pa.: Mosby; 2004. 
Mansel RE, Webster DJT, Sweetland, HM. Hughes, Mansel \& Webster's Benign Disorders and Diseases of the Breast. $3^{\text {rd }}$ ed. Philadelphia, Pa.: Saunders; 2009.

Mayeaux EJ, Cox JT. Modern Colposcopy: Textbook and Atlas. 3rd ed. Philadelphia, Pa.: Lippincott Williams and Wilkins; 2012.

Melmed S, Polonsky KS, Larsen PR, Kronenberg HM. Williams Textbook of Endocrinology. 13 ${ }^{\text {th }}$ ed. Philadelphia, Pa.: Elsevier; 2016.

\section{Website Resources}

American Society for Colposcopy and Cervical Pathology (ASCCP). www.asccp.org/

Centers for Disease Control and Prevention (CDC). www.cdc.gov

Centers for Disease Control and Prevention. US Medical Eligibility Criteria (US MEC) for Contraceptive Use, 2016.

www.cdc.gov/reproductivehealth/contraception/mmwr/mec/summary.html

National Osteoporosis Foundation. www.nof.org/

Reproductive Health Access Project. www.reproductiveaccess.org/

Training in Early Abortion for Comprehensive Healthcare. http://www.teachtraining.org/

U.S. Department of Health and Human Services Office on Women's Health.

www.womenshealth.gov/

U.S. Preventive Services Task Force Recommendations for Primary Care Practice. www.uspreventiveservicestaskforce.org/Page/Name/recommendations

Published: 04/94

Revised: 02/97

Revised 01/04

Revised 01/08 by University of Mississippi Family Medicine Residency Program and the Society of

Teachers of Family Medicine (STFM) Group on Oral Health

Revised $11 / 12$ by Family Medicine Residency of Idaho

Revised/Retitled 06/14 by Bayfront Health St. Petersburg Family Medicine Residency, FL

Revised 08/16 by Contra Costa Family Medicine Residency, Martinez, CA

Revised 08/18 by Sutter Health Family Medicine Residency Program, Sacramento, CA 